\title{
An IoMT Model Based Smart Health Advisory System
}

\author{
K. Asish Vardhan \\ Department of Computer Science \& Engineering, AUCE, Andhra University, \\ Visakhapatnam, India
}

\begin{abstract}
The Internet of Things (IoT) provides Associate in treatment economical and fresh existence to the health care ground. It conjointly includes a speedy development of the many fields. However, many requirements are existing for human health within the field of Medical. One among the advanced move toward doctors can definitely and rapidly correct to employ the pertinent tolerant information's and jointly with the enduring casing the past. Through the Internet of Things, staggeringly improves the normal of data and therefore, the patient heed within the medicinal ground. So, Web of Things offers Associate in Nursing actual platform to interconnect all the resources. Associate in nursing ontology-based mostly automating style methodology for good drugs and physical health system mistreatment IoT. Semantics and ontology mechanisms aid the computers in addition to the considerate the indication and medicinal possessions. So, ontology mechanism assists in making a healing plan and reconfiguring medicinal possessions consistent with the patient's specific necessities apace and repeatedly.
\end{abstract}

Keywords: IoT, Health data, Ontology, Worldwide ontology, Knowledge base, Patient data, Output prescription

\section{Introduction}

By the utilization of IoT challenges square measure rehabilitate, that consumes longer, resources and workforce. At this, we tend to face several challenges within the universe that ought to deal realistically. In recent years the technology and its support to various applications had grown a lot. The utilization of new technology with the help of various web resources and other applications, the development in various areas of focus in the smart health facilities being created and provided to the public in the society had grown a lot. With these, the development of home applications with health monitoring sensors and devices had been increased a lot for both manufacturing and utilization of those sensors. The concept of smart home and smart devices applications will be getting developed a lot in recent years, and especially the involvement of such devices had increased and grown a lot.

With the existing technologies in various areas, the current development in the area of smart data and smart devices utilization for health, home and other resources will be more useful to the public. The utilization of these applications and devices had grown very high and fast in these days. The medical applications had become very costly in the olden days, now with the existing and formatting of these new technologies, the lives of the people will become more comfortable and more accessible for the public to live. Several devices and

Article history:

Received (February 25, 2020), Review Result (March 30, 2020), Accepted (May 5, 2020) 
several applications and other new methods of treatment in medical applications had developed for the current days with the use of these technologies, and these technologies are taking the facilities and provision to the next level of sophisticated life of the patients. The people no need to spend more amount of money for their treatment in the future and also by the usage of these technologies the disease identification had become very easy, i.e. the presence of various symptoms in the human body can be identified by these sensors in the models [1][2].

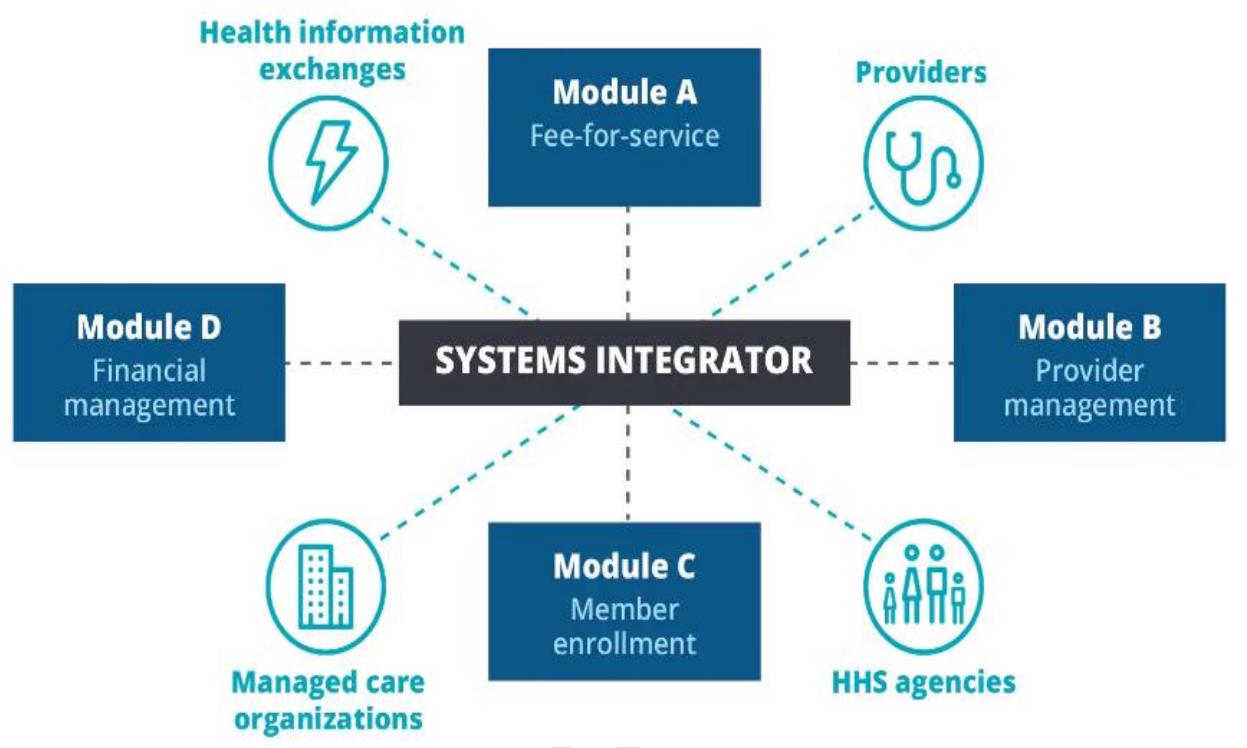

Figure 1. An online smart health model example

Internet of Things (IoT) has several varieties of applications together with care and industrial systems. So, care systems chiefly exploitation the interconnected devices to form associate degree IoT network ardent to assessment, mechanically sleuthing things and monitors the patients, wherever the medical interferences square measure necessary. So, IoT alone will become a kind associate degree info network that interconnects hospitals, peoples, care devices, home atmosphere and different workstations. Implementation of the net of Things is versatile and accessible results to permitting the care applications to serve patients with higher treatment, additionally through with the remote patient watching and effective medical information handling. Their square measures several completely different characteristics square measure required to implement the care service within the atmosphere. However, many requirements are existing for human health within the field of Medical [3].

\subsection{Ontology assessment}

This mechanism works with ontology identification and working on them. The main works done here was that the inputs were collected and analyzed. After that, the collected inputs are processed further for a better analysis of the input symptoms. The disease was primarily identified or assumed, and the data was being sent for further analysis or further checking by the senior experts in the same area of work. The data of the patient also will be logged and stored in the System and also the same data can be shared to the near and far hospitals who can use the same data further for data analysis of patients and also to the Patients arrival to such hospitals. Once, the data of the patient is made standard for all the hospitals and their 
databases, the practical reports and other data can be used by those doctors and lab persons as the previous case and can be studied whenever they want or they use to study the case for better understanding of the cases or for providing the better solutions and medicine sot with the patients [4].

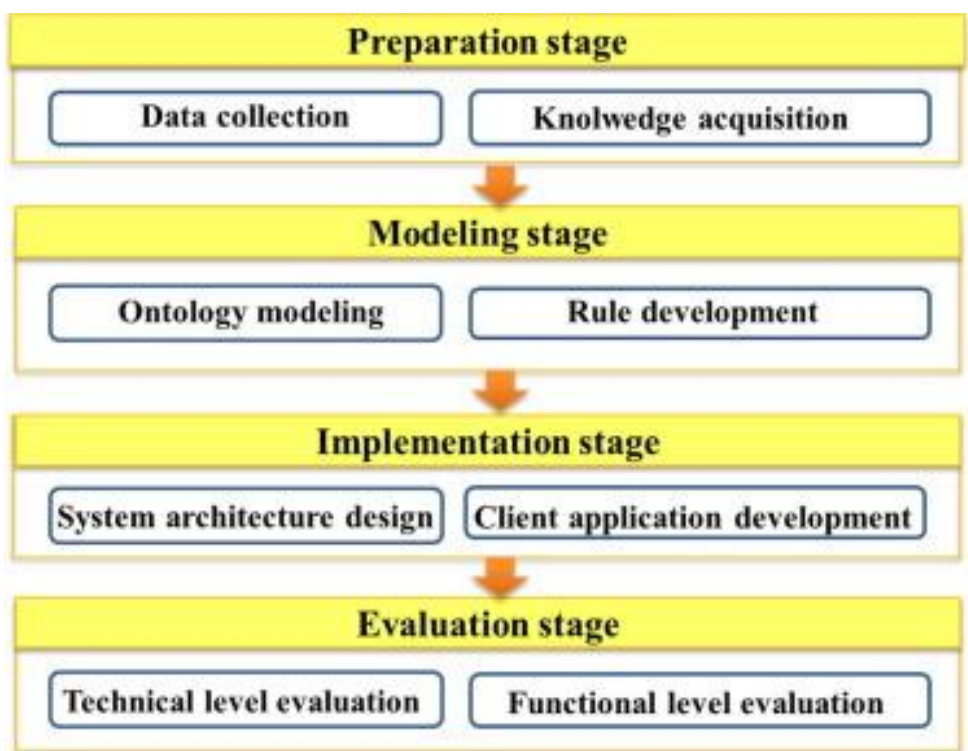

Figure 2. An ontology assessment example

For implementing such technologies and measures for various models, the challenges being faced for these technologies and functions can also be noted and considered seriously [5]. The development of IoT had provided various challenges to the public in society in various countries will be useful. The research on this area is also growing day to day such that the more medical facilities will be provided to more numbers of public ho ewer poor, who cannot afford the costly medicine and also the people who cannot travel more distances for getting the minimum need of quality treatment. Hence, people should support these technologies more and more in such a way to grow more and to develop a mode number of inventions and technology growth in the area of medical applications.

\subsection{Universal ontology evaluation}

Once the patient initial enter the hospitals, the physical characteristics of the diseases are going to be primarily determined by the doctor. The determined functions are divided into the categories and subcategories [6][7][8].

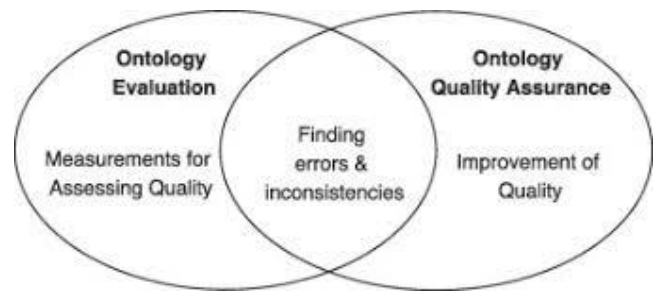

Figure 3. An ontology assessment evaluation example 


\section{Proposed system}

In the projected System, associate ontology primarily based automating style methodology for sensible medication and physical health system mistreatment IoT. In addition to grasping the symptoms and therefore, the medical assets by the utilization of associate ontology. And additionally, associate ontology supports to form a rehabilitation strategy and additionally to reconfigure the medical assets supported the specific needs of the patients routinely and speedily. So, supported the objectives IoT aims to interconnect all the resources and simply give an instantaneous info interaction. The essential plan of IoT is that the pervasive presence of things and objects that square measure interconnected and square measure able to get together with one another to achieve a standard goal.

However, many requirements are existing for human health within the field of Medical. One among the higher approach the doctors are capable to definitely and quickly right to use the relevant patient information's and together with the patient case history. Semantics and ontology mechanisms aid the computers in addition to understanding the symptoms and medical resources.

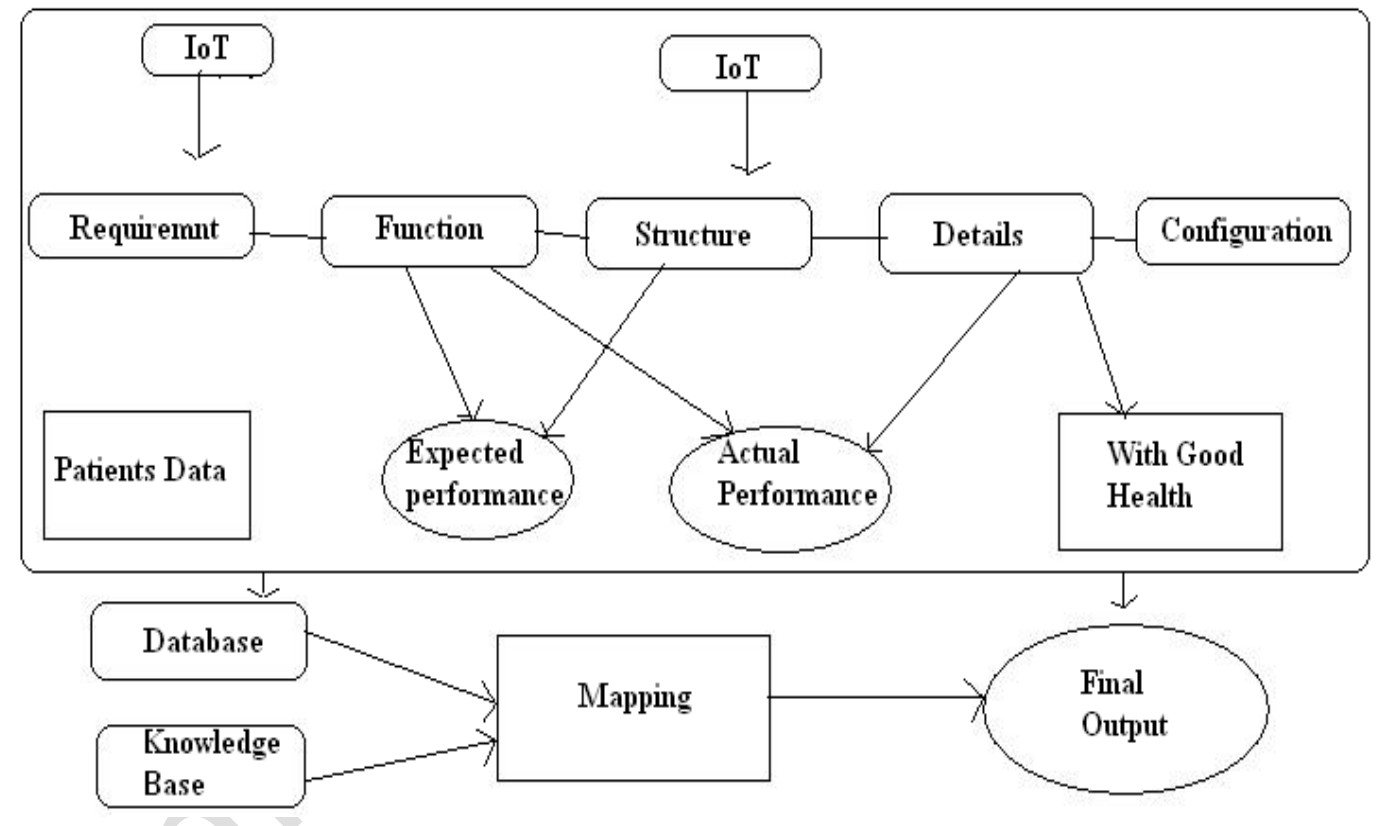

Figure 4. Architecture model of the proposed system

\subsection{Generation of automated design}

The generation of automated style technique ought to be having a number of the stages explained within the implementation and connected works. Once the patient or doctors input the symptoms of the diseases, it is often compared to the world ontology supported mental object. It chooses their most similar case within the mental object. The consumption of medicinal resources should be used correctly. Supported the preparation of scheme is effectual and might make it easy for the synthetic drugs scheme by generating the recommendation. When the automatic style methodology mapping to categories and subcategories of the already keep patients records. 


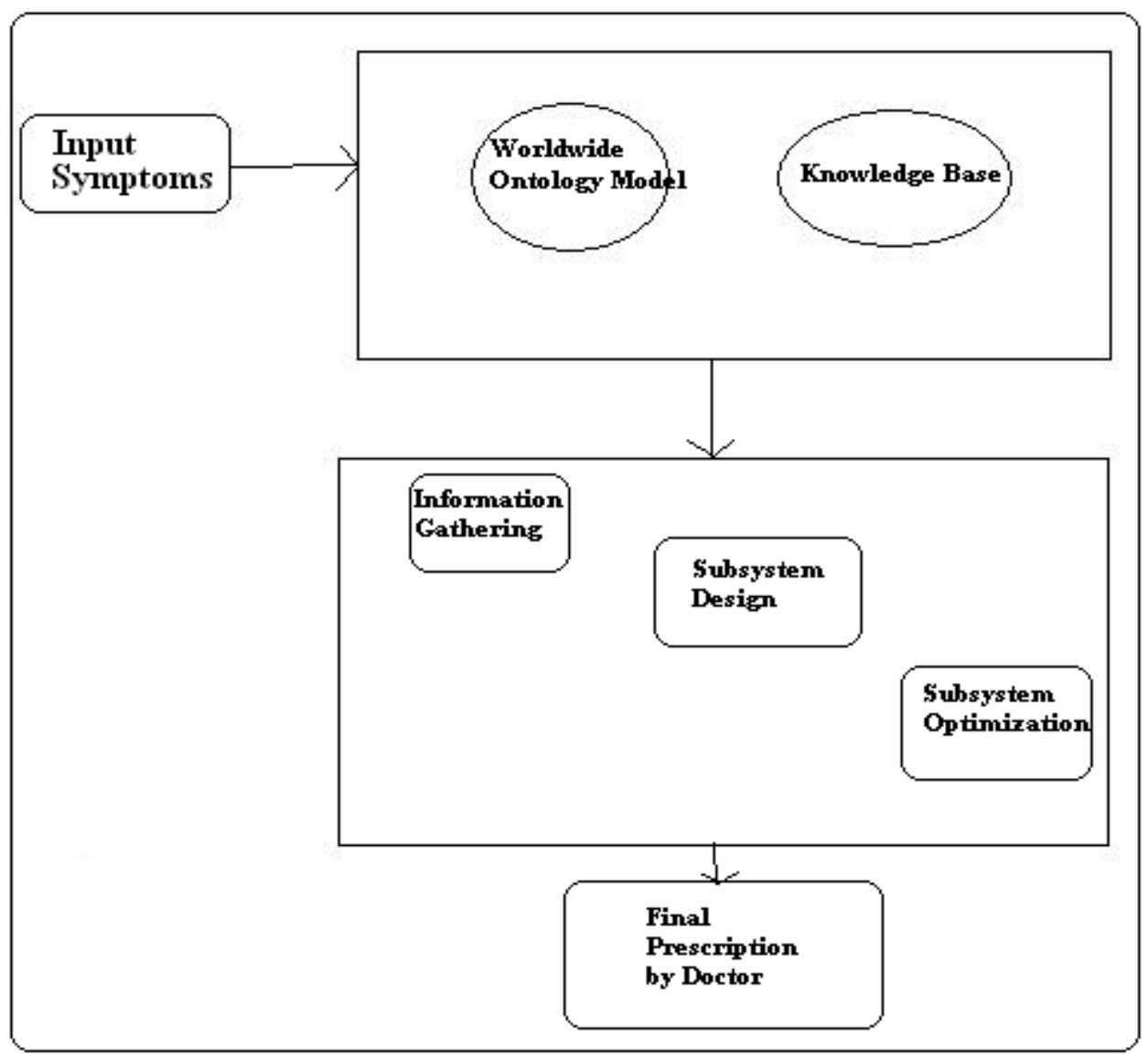

Figure 5. Data generation model of the proposed system

The present scheme will optimize the patient's records supported their diseases and fundamental data. Finally, style collaboration will take a significant role within the style theme to supply the prescription to the patient when the verification of the doctors. By victimization, this technique is often useful for the medical and care systems.

\section{Results and discussion}

The performance of this will be exaggerated to additional economical once compare to the prevailing System. The utilization of medicinal resources ought to be used correctly. Supported the look of the organization is effectual and would perhaps make it accessible to the wise medicine system by generating the recommendation. To understand the proposed model's performance when compared with the previous models, the data and the performance was a little bit good and impressive. For better understanding and better analysis of the work model, the data had been collected with a various set of patients and their symptoms for various diseases and also for the cases to be studied further and can be sent to the furthermore number of doctor experts such that the identification of disease can be made more accessible. 


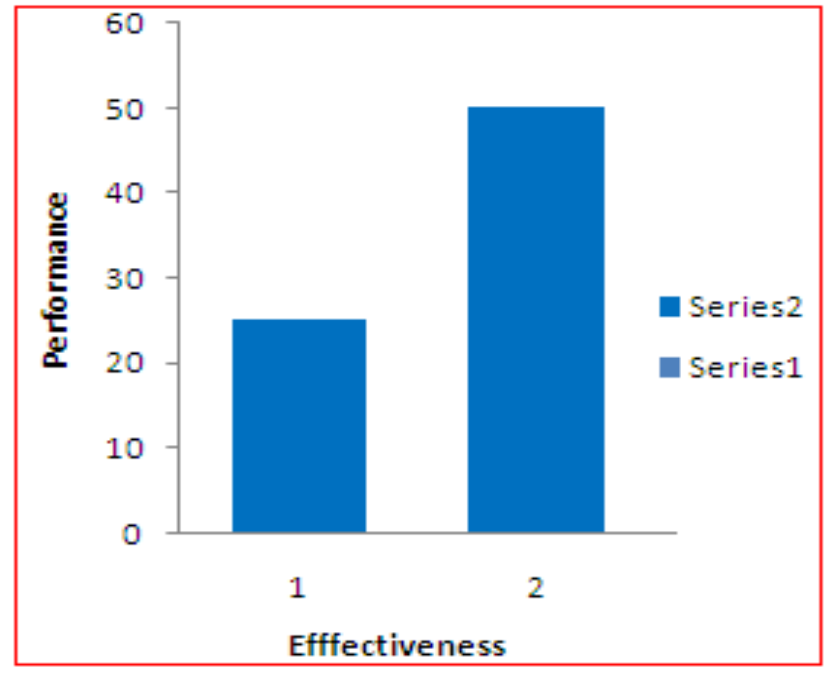

Figure 6. Existing system performance

To understand the performance of the currently considered model, an attempt has been made to identify the performance of the previous existing model for a similar type of datasets those were considered in the current model. As a result of this, the performance had observed, and it had represented in the form of graphical representations, and the data was already shown in figure 5. The series shown in [Figure 5] was that the data collected in two phases and the data was made with two sets. The model was implemented, and the results were represented in the above [Figure 5].

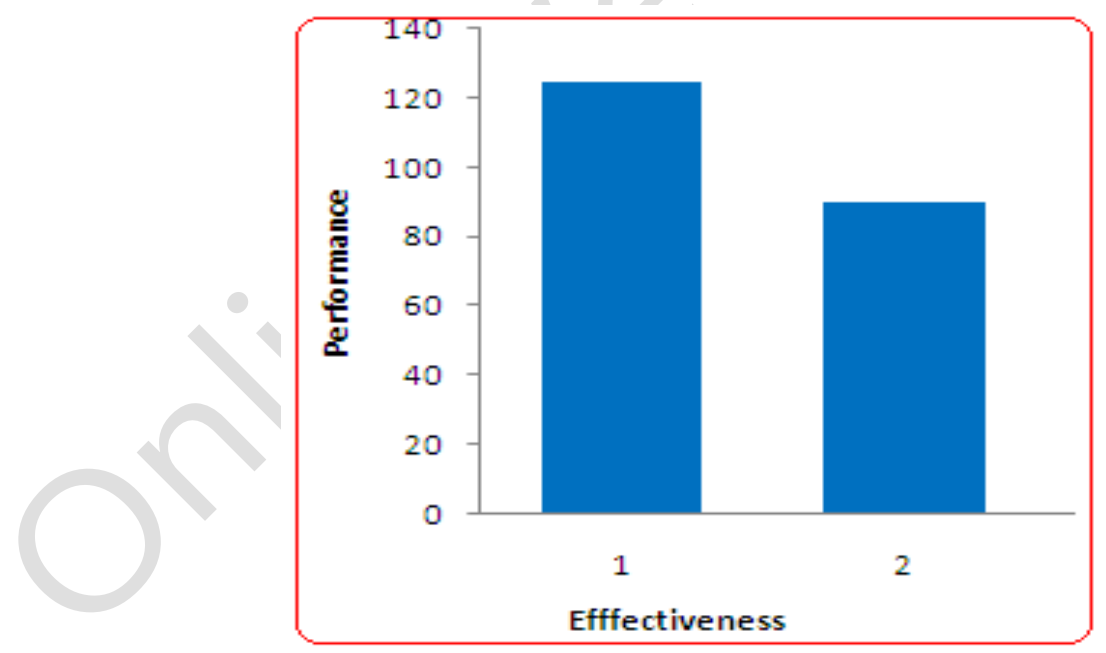

Figure 7. Proposed system performance

Once the patient or doctors input the symptoms of the diseases, it is often compared to the world ontology supported mental object. It chooses their most similar case within the mental object. The performance of this will be exaggerated to additional economical once compare to the prevailing System. The other points considered here are the implementation of optimization models in the current model such that to optimize the identification of diseases 
with the currently provided symptoms and also to play a vital role in disease identification. The prescription to the patient through online also being generated and also be sent to the patient in the form of either a text message or some email. The patient's records also will maintain through online, and those records can be able to access to anywhere in the world for any previous case studies or any reference. The records can be maintained in the form of patient's gender, area of stay, country of stay, the age and other details of the patients such that analysis of the patient's data can be made more comfortable. The understanding of the patient details will be made more comfortable and also made available to anywhere it he world. The doctors can access this data not only through some laptops or desktop machines but also through mobile phones also can access such data and can e useful for their future patient's data analysis and disease identification to any patient with a similar set of symptoms.

\section{Conclusions}

Health is more important for any sort of human being in the society irrespective of their gender or their financial status. Some people with an excellent financial background can have costlier medicine and some other with less money cannot near the costlier functional medicine. The other serious problem is that the people living in rural areas cannot be reached with high-end excellent health facilities. As a result, most of the people in rural areas are getting affected by serious health issues, and they are not able to reach to medical facilities too. In the current application, an attempt had been made to provide a facility of proper medical facilities and proper treatment to the patients living in rural areas. The current application had supported the planning of System is effective and might facilitate the sensible drugs system by generating the prescription. This technique should be each effective and economical for the data sharing. Two necessary options, as well as the simple allocation of area information that ought to build the System as characteristic and execute estimable, as IoT and ontology have contended a vital role within the methodology.

\section{References}

[1] D. Yongsheng, J. Yanling, R. Lihong, and H. Kuangrong, "An intelligent self- organization scheme for the Internet of Things," IEEE Computer Intelligence Magazine, vol.8, no.3, pp.41-53, (2013)

[2] L. Meghan, "IoT livestock estrus monitoring system based on machine learning," Asia-pacific Journal of Convergent Research Interchange, vol.4, no.3, pp.119-128, (2018) DOI:10.14257/apjcri.2018.09.12

[3] Majerol M., Carroll W., and Fingar S., "Smart Medicaid, Deloitte Insights," accessed 19 March 2020, https://www2.deloitte.com/us/en/insights/industry/health-care/smart-technology-in-health-care-medicaidprograms.html, (2018)

[4] P. Sandeep Kumar, "IoMT based smart health care monitoring system," International Journal for Innovative Research in Science \& Technology, vol.5, no.11, pp.58-64, (2019)

[5] Z. Yi Fan, G. Ling, Z. Tian Shu, L. De Nan, Z. Jing, L. Ye, and L. Li Song, “An ontology-based approach to patient follow-up assessment for continuous and personalized chronic disease management," Journal of Biomedical Informatics, vol.72, pp.45-49, (2017)

[6] P. Sandeep Kumar, "NFC based smart healthcare services system," International Journal for Innovative Research in Science \& Technology, vol.5, no.7, pp.45-48, (2018)

[7] A. Muhammad, H. Zhe, B. Jiang, L. Juan Antonio, and T. Cui, "Assessing the practice of biomedical ontology evaluation: Gaps and opportunities," Journal of Biomedical Informatics, vol.80, pp.1-13, (2018)

[8] M. Chiara, "Plate element formulation for a discrete element method," International Journal of Advanced Science and Technology, vol.94, pp.13-22, (2016) 
An IoMT Model Based Smart Health Advisory System

This page is empty by intention. 